\title{
Durable Clinical Response to Immune and Targeted Therapies in an Elderly Man with Synchronous Gastric (HER2+) and Bladder Cancers: Case Report and Literature Review
}

Xin Tian

Qiuxia Zhu

Zhenyong Zhang

Department of Oncology, Shengjing Hospital of China Medical University, Shenyang, Liaoning, I I0022, People's Republic of China
Correspondence: Zhenyong Zhang Department of Oncology, Shengjing Hospital of China Medical University, Shenyang, Liaoning, I I0022, People's Republic of China

Email zhangzhenyongvip@163.com
Abstract: Synchronous occurrences of gastric cancer positive for human epidermal growth factor receptor 2 (HER2+) and bladder cancer are rarely encountered in clinical practice. When and how to effectively treat both tumors, without compounding adverse effects, must be addressed. Herein, we describe an elderly man who presented with both gastric cancer (HER2+) and bladder cancer. Due to enlarged and fused lymph nodal metastasis, he was illsuited for stomach resection. After transurethral resection of the bladder tumor, we administered both chemotherapy and the targeted agent trastuzumab. Gastric cancer showed partial response however bladder cancer recurred following two cycles of this regimen, the adverse effects were prohibitive, prompting refusal of further chemotherapy and radiotherapy. $\mathrm{He}$ then received the immune checkpoint inhibitor (ICI) nivolumab and trastuzumab in combination. This particular regimen successfully controlled both cancers and substantially improved the patient's quality of life. Its long-term use did not intensify adverse reactions, enabling a progression-free survival of 21 months to date. We have also reviewed other published clinical strategies applied in rare instances of multiple primary malignancies as a reference for treating such patients.

Keywords: immunotherapy, targeted therapy, synchronous cancer, HER2-positive gastric cancer, bladder cancer

\section{Introduction}

Multiple primary malignant tumors (MPMTs) developing in the same individual may be synchronous or metachronous. A majority of multi-organ MPMTs are metachronous, whereas synchronous MPMTs are far less common. ${ }^{1}$ Patients with synchronous gastric and bladder cancers, including gastric tumor positivity for human epidermal growth factor receptor 2 (HER2+), are rarely seen in clinical practice, so large-scale clinical studies and established therapeutic guidelines are lacking. Treatment usually depends on the staging of each tumor, their specific histotypes, and the patient's physical condition. A multidisciplinary and patientoriented approach is best, helping to decide when and how to effectively treat both cancers without compounding known adverse effects

Immune and targeted therapies have increasingly become the most promising antitumor strategies in use. ${ }^{2-5}$ Immunotherapy has shown satisfactory results in treating both gastric and bladder cancers, ${ }^{6,7}$ and through the targeting of HER2, 
trastuzumab offers better progression-free (PFS) and overall (OS) survival for patients with HER2+ gastric cancers. ${ }^{8,9}$ Many preclinical studies and clinical research efforts conducted in recent years have proven the utility of combining immune and targeted therapies, reporting improved therapeutic responses and prolonged survival times in patients with many types of cancers. ${ }^{10-12}$ Although each agent separately has previously shown efficacy for HER2+ gastric cancer, combined usage has yet to be reported, and there are no prior accounts of dual-agent use for two different tumors in the same patient.

Herein, we present an elderly man with synchronous gastric cancer (HER2+) and bladder cancer who clearly benefited from long-term combined use of immune (nivolumab) and targeted (trastuzumab) agents. This is the first known report of its kind, addressing efficacy and safety aspects of a nivolumab/ trastuzumab regimen given to an aged patient with two synchronous cancers. Our intent was to explore strategies for evaluating and managing similar scenarios, based on our clinical experience and that reported by others.

\section{Case Presentation}

During November 2018, a 73-year-old Chinese man was stricken with stomach pain and gross, painless hematuria. In January of the following year, he was admitted to our hospital's urology department for cystoscopy and gastroscopy. Gastroscopy revealed an ulcerative lesion $(4 \mathrm{~cm}$ $\mathrm{x} 1.5 \mathrm{~cm}$ ) of gastric horn (Figure 1A), diagnosed as gastric adenocarcinoma. Signet ring cells were visible in histologic sections (Figure 1B), and the following tissue markers exhibited immunostaining positivity: MLH1 (+), MSH2 $(+)$, MSH6 (+), PMS2 (+) (Figure 2A-D), and HER2 (2 $+)$. HER2 amplification was also evident by fluorescence in situ hybridization (Figure 1C); multiple enlarged and fused tumor-bearing lymph nodes appeared in computed tomography (CT) of the abdomen, rimming the stomach (Figure 3A and B). Cystoscopy and abdomen CT also disclosed multiple bladder tumors (Figure 1D and E) (Figure 3C) histologically confirmed as urothelial carcinoma (Figure 1F). A multi-disciplinary team (MDT) convened to discuss feasible treatment options. Considering
A

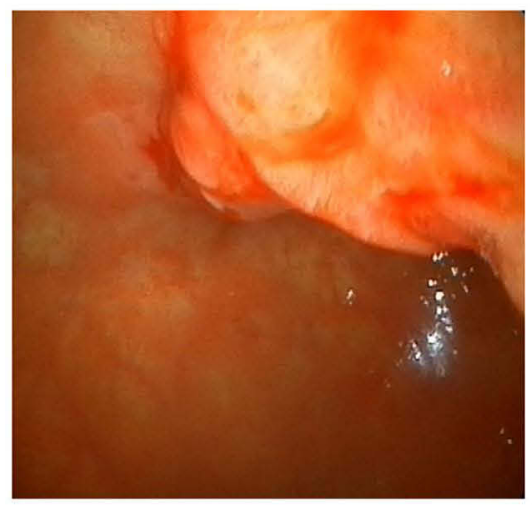

D

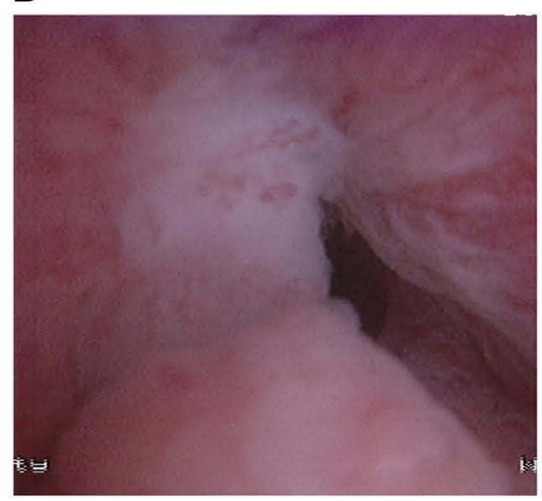

B

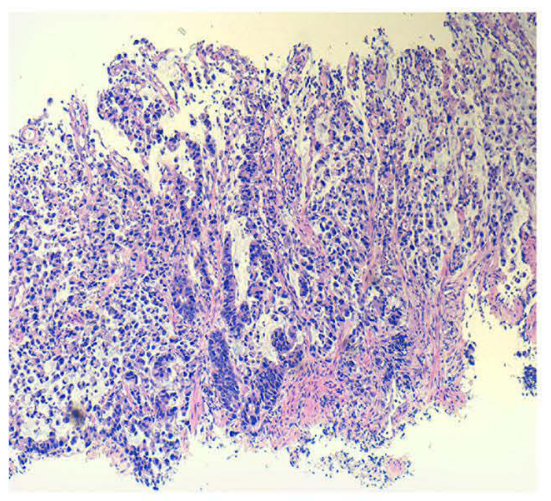

E

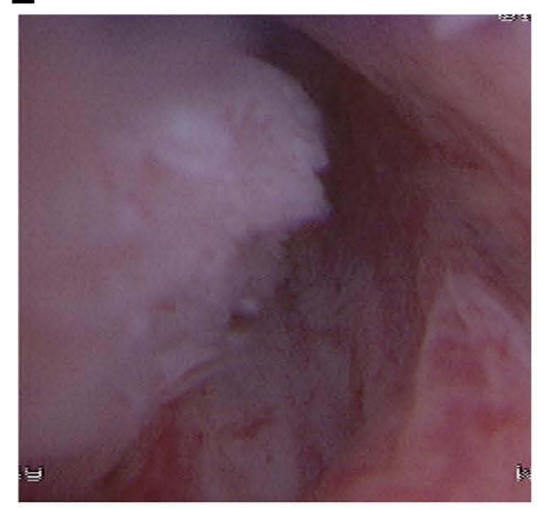

C

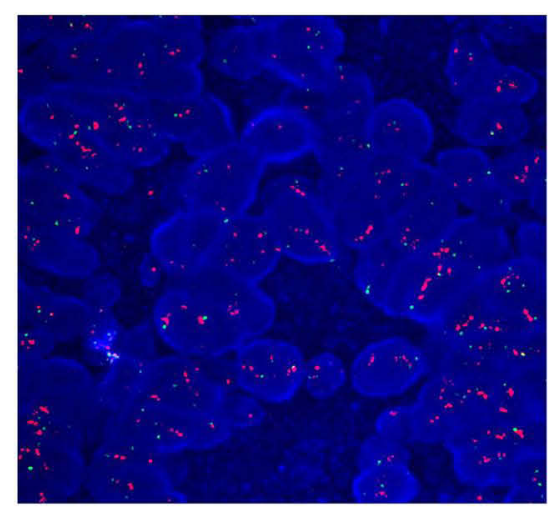

$\mathbf{F}$

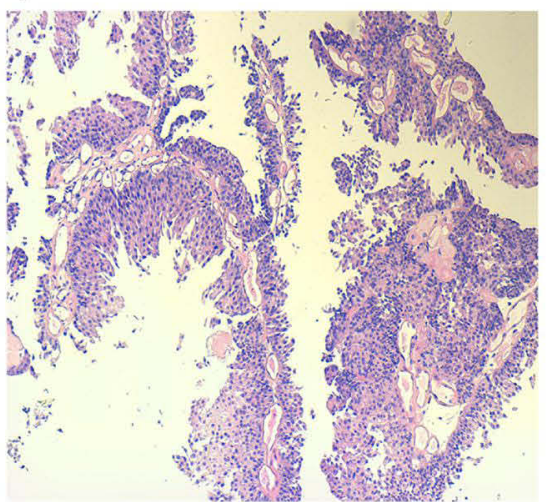

Figure I Gastroscopy, cystoscopy and histopathological results of the patient. (A) Gastroscopy revealed an $4 \mathrm{~cm} \times 1.5 \mathrm{~cm}$ ulcerative lesion in the gastric horn. (B) Histopathologic diagnosis was gastric adenocarcinoma, signet ring cell carcinoma can be seen microscopically. (C) In Situ Hybridization (FISH) showed HER2 amplification. (D and E) Cystoscopy revealed multiple bladder masses. (F) Histopathologic diagnosis was urothelial carcinoma. 

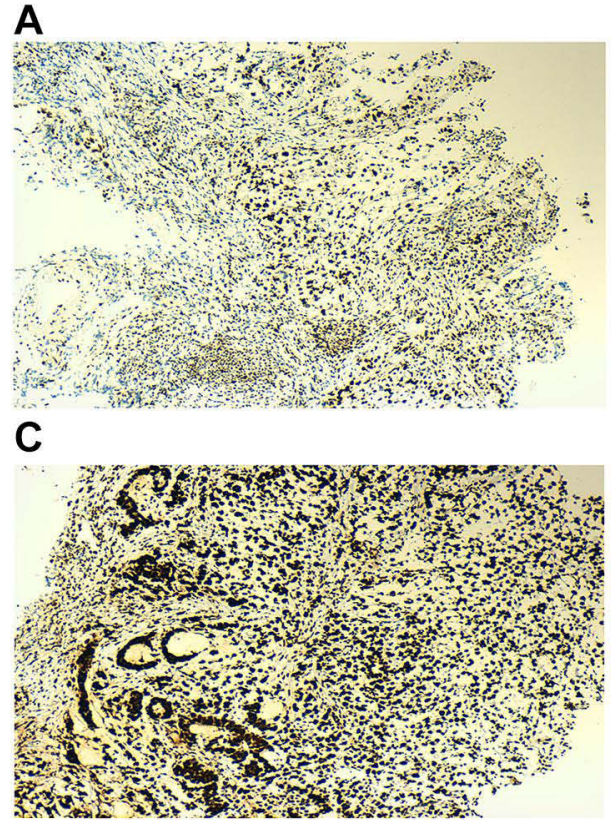

E

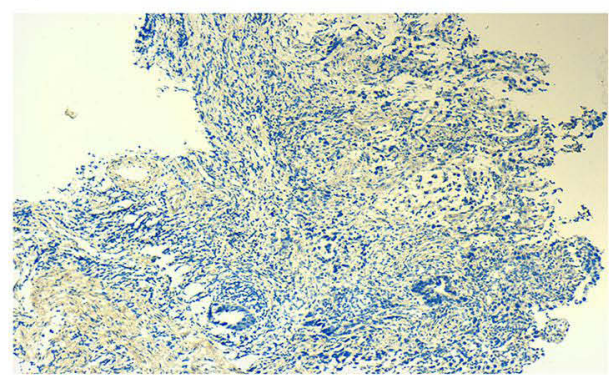

B

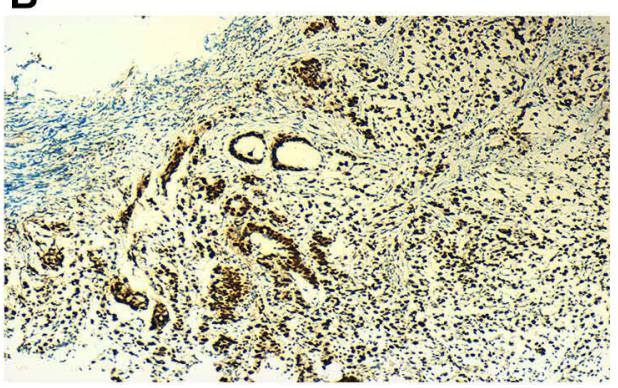

D

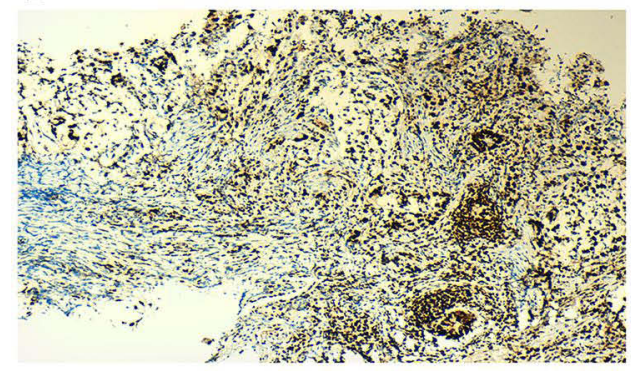

$\mathbf{F}$

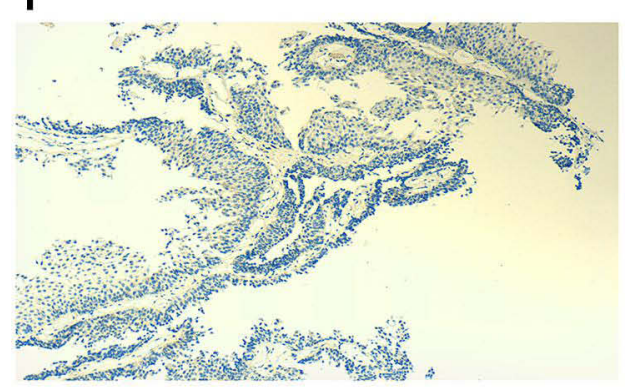

Figure 2 Immunohistochemical results of the patient. (A) MLHI (+). (B) MSH2 (+). (C) cancer.

the metastatic lymph nodes with multiple enlargement and fusion around the stomach may not be completely cleared by surgery, the advanced age of the patient and the pathological type of signet-ring cell carcinoma with high malignancy, our gastrointestinal surgeon did not recommend surgical treatment. So transurethral resection of the bladder cancer took place on February 14, 2019 in the urology department considering the patient's will of preserving the bladder. Postoperative recovery was good, but the preserving bladder surgery placed this patient at high risk of recurrence and progress.

\section{Combination Chemotherapy Plus Trastuzumab}

Between February and April of 2019, the patient received two cycles of SOX (S-1 plus oxaliplatin) combination chemotherapy, given once every 3 weeks, in conjunction with intravenous trastuzumab. He also received six cycles of once-weekly gemcitabine-only bladder perfusion. CEA and CA-724 levels were decreased during this time (Figure 4A and B), abdominal CT for gastric cancer showed some shrinkage of the enlarged perigastric lymph nodes (Figure 3D and E). However, abdominal CT indicated new tumor in bladder and a recurrence of bladder cancer was considered (Figure 3F). Unfortunately, low patient tolerance (ie, nausea, vomiting, and myelosuppression) prohibited of an otherwise effective chemotherapeutic protocol. A MDT convened to discuss feasible treatment options again. Secondary resection of the recurrent bladder cancer, radiotherapy and next-generation sequencing was then advised by doctors, but the patient refused. We subsequently obtained PD-L1 immunostains for both tumors, finding them negative. 
A

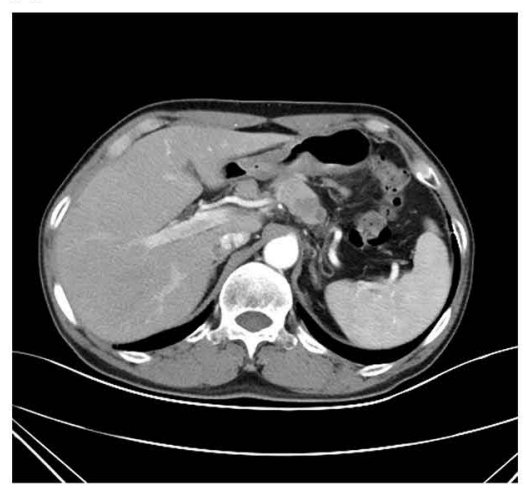

D

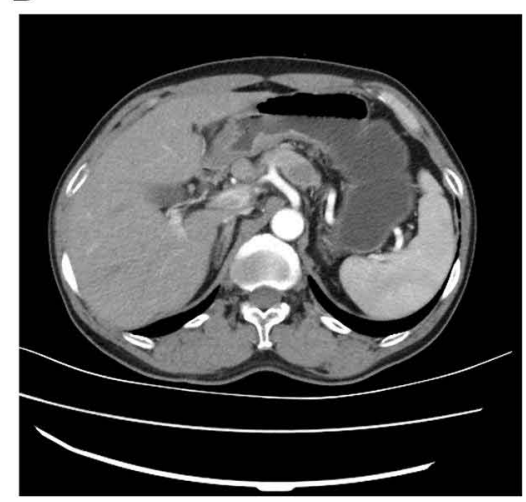

G

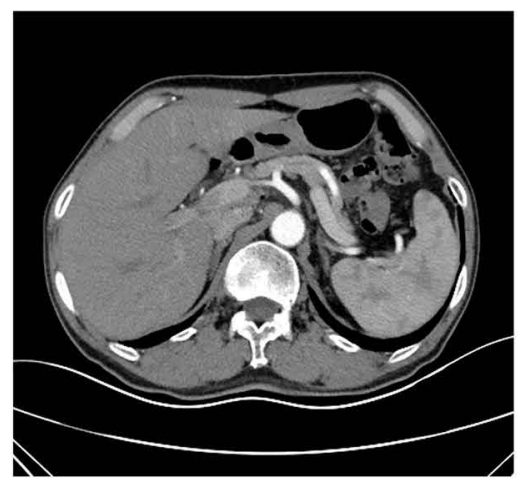

B

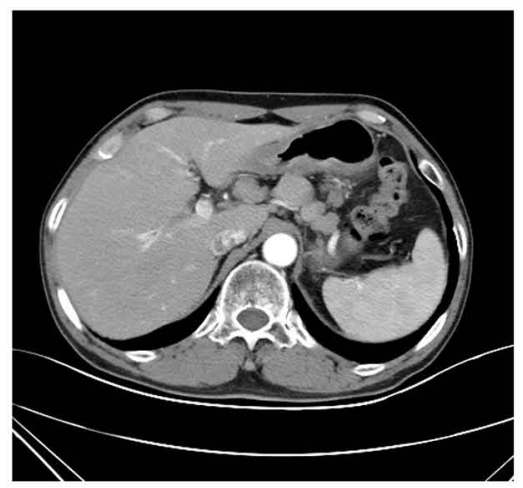

E

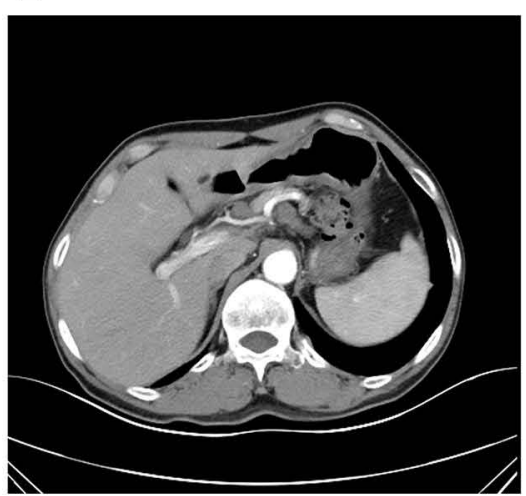

H

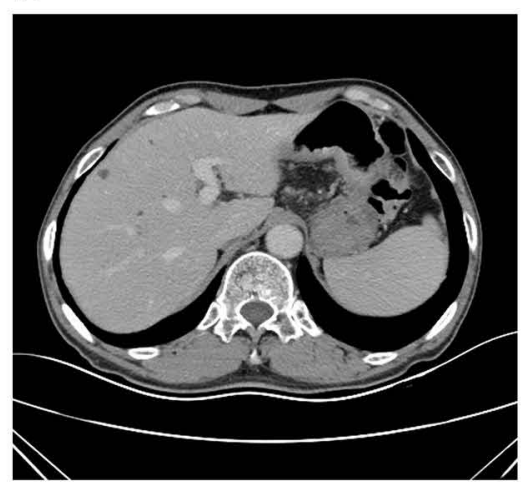

C

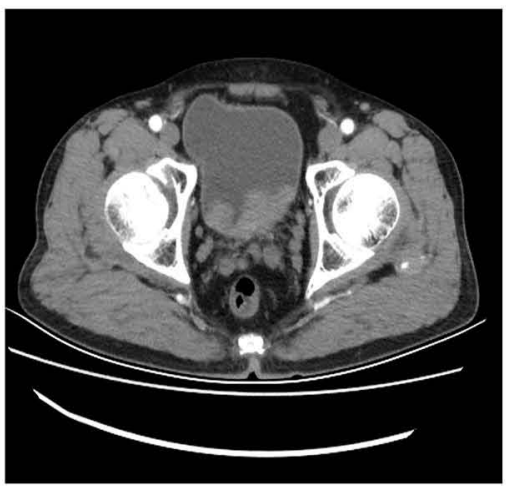

$\mathbf{F}$

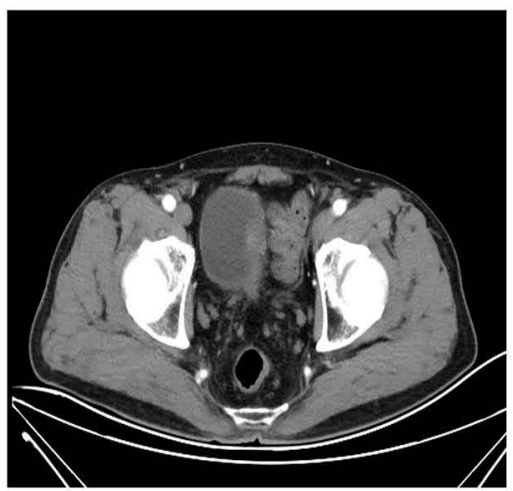

I

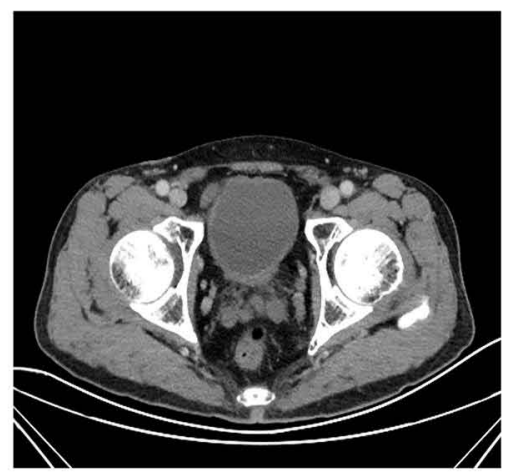

Figure 3 Abdominal CT images of the patient. (A and B) Abdominal CT images at initial diagnosis indicated multiple enlarged lymph node metastases around the stomach. (C) Abdominal CT images at initial diagnosis indicated multiple bladder tumors. (D and E) Abdominal CT images after 2 cycles of chemotherapy indicated multiple enlarged lymph node metastases around the stomach were smaller. (F) Abdominal CT images indicated new tumor in bladder and a recurrence of bladder cancer was considered. ( $\mathbf{G}$ and $\mathbf{H}$ ) Abdominal CT scan after 8 cycles of immunotherapy plus targeted therapy showed a significant reduction and shrinkage of metastatic lymph nodes around the stomach which suggested a partial response of the gastric cancer. (I) Abdominal CT scan showed the recrudescent bladder cancer was significantly shrunk after 8 cycles of immunotherapy plus targeted therapy.

\section{Nivolumab/Trastuzumab Regimen}

The PD-1 inhibitor nivolumab (3 $\mathrm{mg} / \mathrm{kg}$, Q2W) combined with trastuzumab began in May 2019. Two months after initiating this regimen, a rise in transaminase occurred but was normalized through use of one week of liverprotective medicine Magnesium Isoglycyrrhizinate Injection. The patient received a general examination every 3 months during treatment. After eight cycles of immunotherapy, abdominal CT showed a significant reduction and shrinkage of metastatic lymph nodes around the stomach which suggested a partial response of the gastric cancer (Figure $3 \mathrm{G}$ and $\mathrm{H}$ ). Abdominal $\mathrm{CT}$ also confirmed the recrudescent bladder cancer was significantly shrunk (Figure 3I). CEA and CA-724 levels were also decreased 


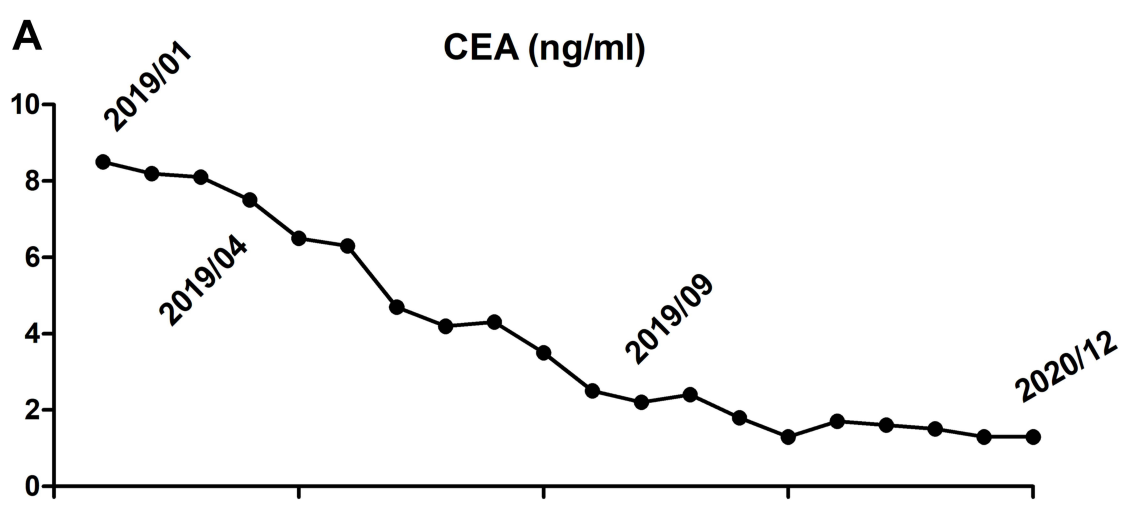

B $\quad$ CA-724 (U/ml)

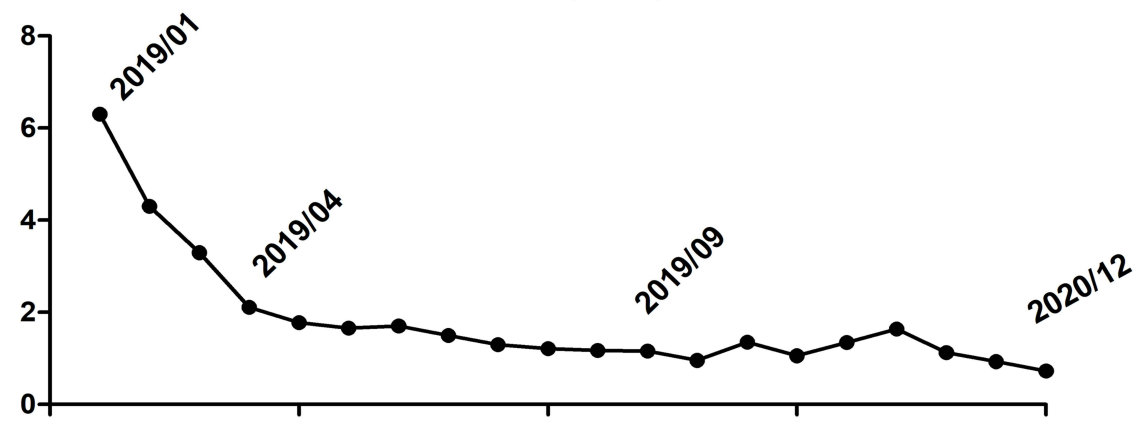

Figure 4 Cancer CEA $(\mathrm{ng} / \mathrm{mL})$ and CA-724 $(\mathrm{U} / \mathrm{mL})$ levels of the patient. (A) CEA levels during entire treatment. (B) CA-724 levels during entire treatment.

(Figure 4A and $\mathrm{B}$ ), and the patient's quality of life improved substantially. This regimen is ongoing at present, and the patient's condition continues to be stable The progression-free survival (PFS) was 21 months since he received the combination treatment.

\section{Discussion}

MPMTs are rare among patients with malignant tumors. In recent years, the aging population has grown as diagnostic methods and anti-cancer therapies continue to advance, so similar reports of multiple tumors at differing sites are surfacing. ${ }^{13,14}$ Environmental factors, aging, unhealthy lifestyles, cancer treatments, or interactions thereof may spur the development of MPMTs. Unlike isolated occurrences, the pathogenesis of multiple malignancies may be linked to specific genetic defects detectable by nextgeneration sequencing. ${ }^{15}$ Still, standard treatments for MPMTs are not well established. Parameters to consider in treatment planning include certain tumor-specific (stage, biologic behavior) and patient-related variables (age, physical condition, life expectancy, and comorbidities).

In clinical practice, it is important to distinguish between multiple primary malignancies and a single primary with metastasis. Because neither stomach nor bladder is prone to metastasis to each other, we anticipated two synchronous primary cancers on this occasion, which indeed was histologically substantiated. We then engaged a MDT to formulate individualized treatment, calling upon departments of gastrointestinal surgery, urology, radiotherapy, oncology, imaging, and pathology to contribute their expertise. This approach is critical in the context of malignant diseases, especially MPMTs, helping to optimize therapeutic regimens. Ultimately, we abandoned gastric resection due to enlarged and fused lymph nodal metastasis. We then proceeded with transurethral resection, as stipulated by National Comprehensive Cancer Network (NCCN) guidelines for treatment of bladder cancer and the patient's will, followed by gemcitabine bladder perfusion for bladder cancer and an intravenous $\mathrm{SOX}+$ trastuzumab regimen for gastric cancer. In patients with advanced HER2+ gastric cancers not amenable to surgery, better PFS and OS are reportedly achieved by combining trastuzumab with chemotherapy. ${ }^{16}$ Perfusion chemotherapy is presently the chief postsurgical means of treating bladder cancer, ${ }^{17}$ and the SOX combination was chosen for its better tolerability in patients too old for rigorous chemotherapy. Similarly, the choice of gemcitabine (vs 
Bacillus Calmette-Guerin) for bladder perfusion was dictated by its better tolerability. After two cycles of chemotherapy, gastric cancer showed partial response however bladder cancer recurred, the related adverse effects were severe. The treatment regimens are often lead to greater toxicity for patients with MPMTs. When and how to effectively treat both cancers without undue toxicity must be addressed.

The introduction of immune checkpoint inhibitors (ICIs) during recent years has brought improvements in treating advanced gastric and bladder cancers. ${ }^{18-20}$ However, research is lacking on efficacy/safety aspects of ICI use for MPMTs; and despite the proven efficacy of monotherapy trastuzumab and ICIs in patients with HER2+ gastric cancer, we have seen little reporting of related efficacy and safety of the combination use. Also, the long-term survival benefit of this strategy remains unclear. In our patient at least, both primary cancers were well controlled through dual-agent immune and targeted therapy, with substantial gains in quality of life. This was surprising, given that histologic (ie, signet-ring cells, HER2 amplification) and clinical stage indices of his gastric cancer were high grade and prognostically dismal. There is evidence that HER2 protein plays a critical role in the tumorigenesis, progression, and metastasis of HER2+ gastric cancer, imparting a poor prognosis. ${ }^{21}$ Furthermore, after recurrence of the bladder cancer, although the patient refused to receive secondary resection of the bladder cancer or radiotherapy, the tumor still achieved a significant remission. PFS has presently reached 21 months under the ongoing protocol and will be extended. This long-term combined use of nivolumab and trastuzumab has yielded optimal treatment responses by both cancers. However, considering the favourable results of nivolumab for bladder cancer, there is one possibility that the beneficial results for bladder cancer of him may accounted to the effect of the nivolumab monotherapy.

It is debatable whether molecular biomarkers accurately predict immunotherapeutic outcomes. Some studies have found several (eg, PD-L1, TMB, MSI, and HER2) reflective of ICI efficacy, ${ }^{22}$ whereas two prior clinical trials (ONO-4538-12, ATTRACTION-2) have determined that the survival benefit of nivolumab is independent of PD-L1 positivity. ${ }^{23}$ Although both cancers were in fact negative for PD-L1 expression, and microsatellite stable (MSS) for gastric cancer, our patient still benefited from long-term use of a nivolumab/trastuzumab combination. It is reported antibody therapy mediated immune stimulation and PD-L1 blockers, can be exploited to magnify the efficacy of HER2-targeting mAbs along with the elicitation of robust anticancer immunity. ${ }^{24}$ Subsequent in vitro and in vivo experiments are needed to confirm the probable synergism between these two agents.

The safety of combining immune and targeted therapeutics does merit some discussion. After two months of combined treatment, our patient displayed a rise in transaminase level that was reversed and further prevented through hepatic protective drug treatment. Adverse reactions of this sort have been reported when evaluating the efficacy of PD-1 immunotherapy. According to Masuda et al, the immunerelated adverse events that emerge are linked to clinical benefit of nivolumab in patients with advanced gastric cancer. ${ }^{25}$ This seemed to be the case in our patient. It has also been reported that the main adverse reaction to trastuzumab is impaired left cardiac function. ${ }^{26,27}$ However, no such dysfunction occurred in our patient during long-term combination use. It thus appears that extended administration of immune and targeted therapies in combination does not heighten adverse patient reactions.

Our efforts were not without limitations. The chief drawback is that we did not obtain next-generation sequencing of this patient's tumor mutational burden and MSI status for bladder cancer. Another issue is the lack of sufficient follow-up data. Lengthier periods of patient monitoring are needed to confirm the efficacy and safety of this particular therapeutic approach.

\section{Conclusion}

A dual-agent immune and targeted therapeutic regimen administered to treat two synchronous cancers (HER2+ gastric and bladder) in an elderly man yielded optimal treatment responses. Long-term use of nivolumab and trastuzumab seemed effective and safe in this instance, serving as a reference for future treatment of such patients. Further studies are needed to validate this outcome.

\section{Ethical Approval}

All procedures performed in studies involving human participants were in accordance with the ethical standards of the institutional and/or national research committee and with the 1964 Helsinki declaration and its later amendments or comparable ethical standards. The committees of Shengjing Hospital of China Medical University approved to publish the case details. 


\section{Consent for Publication}

The patient provided written informed consent for the case details to be published.

\section{Acknowledgments}

The authors would like to thank the pathologist Xiufang Ren from Shengjing Hospital of China Medical University as well as the patient and his family.

\section{Funding}

The study was funded by the Natural Science Foundation of Liaoning Province (No.20180551038).

\section{Disclosure}

All authors declare no conflicts of interest in this work.

\section{References}

1. Li N, Liu X, Song Y, Luo S, Fang B. The synchronous presence of multiple myelomas and other primary malignant tumors: case series with literature review. Cancer Manag Res. 2020;12:2829-2838. doi:10.2147/CMAR.S238288

2. Sun JY, Lu XJ. Cancer immunotherapy: current applications and challenges. Cancer Lett. 2020;480:1-3. doi:10.1016/j. canlet.2020.03.024

3. Tan S, Li D, Zhu X. Cancer immunotherapy: pros, cons and beyond. Biomed Pharmacother. 2020;124:109821. doi:10.1016/j. biopha.2020.109821

4. Ruiz-Cordero R, Devine WP. Targeted therapy and checkpoint immunotherapy in lung cancer. Surg Pathol Clin. 2020;13:17-33. doi:10.1016/j.path.2019.11.002

5. Kim JH, Park SJ. Target therapy in unresectable or metastatic colorectal cancer. Korean J Gastroenterol. 2016;68:303-311. doi:10.4166/ kjg.2016.68.6.303

6. Qiu H. Safety and efficacy of toripalimab in advanced gastric cancer: a new clinical trial bringing hope for immunotherapy in gastric cancer. Cancer Commun (Lond). 2020;40:194-196. doi:10.1002/ cac2.12019

7. Califano G, Ouzaid I, Verze P, Stivalet N, Hermieu JF, Xylinas E. New immunotherapy treatments in non-muscle invasive bladder cancer. Arch Esp Urol. 2020;73:945-953.

8. Lee SY, Oh SC. Changing strategies for target therapy in gastric cancer. World J Gastroenterol. 2016;22:1179-1189. doi:10.3748/ wjg.v22.i3.1179

9. Franchi M, Tritto R, Torroni L, Reno C, La Vecchia C, Corrao G. Effectiveness and healthcare cost of adding trastuzumab to standard chemotherapy for first-line treatment of metastatic gastric cancer: a Population-Based Cohort Study. Cancers (Basel). 2020;12:1691. doi:10.3390/cancers12061691

10. Naderi-Azad S, Sullivan R. The potential of BRAF-targeted therapy combined with immunotherapy in melanoma. Expert Rev Anticancer Ther. 2020;20:131-136. doi:10.1080/14737140.2020.1724097
11. Rassy E, Flippot R, Albiges L. Tyrosine kinase inhibitors and immunotherapy combinations in renal cell carcinoma. Ther Adv Med Oncol. 2020;12:1758835920907504. doi:10.1177/1758835920907504

12. Wu ZJL, Li K, Zhang K, Gong WD. Research progress of immunotherapy alone and in combination for liver cancer. Zhonghua Gan Zang Bing Za Zhi. 2020;28:471-474. doi:10.3760/cma.j.cn50111320200520-00262

13. Zhao Z, Sun K, Yan T, Wei R, Guo W. Multiple primary tumors: a case report and review of the literature. BMC Musculoskelet Disord. 2020;21:394. doi:10.1186/s12891-020-03426-8

14. De Luca A, Frusone F, Vergine M, et al. Breast cancer and multiple primary malignant tumors: case report and review of the literature. In Vivo (Brooklyn). 2019;33(4):1313-1324. doi:10.21873/invivo.11605

15. Zhang L, Feng L, Cong H, et al. Multiple primary malignant neoplasms: a case report and literature review. Oncol Lett. 2019;18:4210-4220. doi:10.3892/ol.2019.10779

16. Gürbüz M, Akkuş E, Sakin A, et al. Combination of trastuzumab and taxane-containing intensified chemotherapy in first-line treatment of HER2-positive advanced gastric cancer. Tumori. 2020;9:300891620969823. doi:10.1177/0300891620969823

17. Chang SS, Bochner BH, Chou R, et al. Treatment of non-metastatic muscle-invasive bladder cancer: AUA/ASCO/ASTRO/SUO guideline. J Urol. 2017;198:552-559. doi:10.1016/j.juro.2017.04.086

18. Nakajima TE, Kadowaki S, Minashi K, et al. Multicenter Phase I/II Study of nivolumab combined with paclitaxel plus ramucirumab as second-line treatment in patients with advanced gastric cancer. Clin Cancer Res. 2020. doi:10.1158/1078-0432.CCR-20-3559

19. Matsumoto T, Yamamoto Y, Kuriona Y. Efficacy and safety of nivolumab for advanced gastric cancer patients with poor performance statuses. BMC Cancer. 2020;20:684. doi:10.1186/s12885-020-07176-7

20. Wołącewicz M, Hrynkiewicz R, Grywalska E. Immunotherapy in bladder cancer: current methods and future perspectives. Cancers (Basel). 2020;12:1181. doi:10.3390/cancers12051181

21. Chua TC, Merrett ND. Clinicopathologic factors associated with HER2-positive gastric cancer and its impact on survival outcomes-a systematic review. Int J Cancer. 2012;130:2845-2856. doi:10.1002/ijc. 26292

22. Shitara K, Özgüroğlu M, Bang YJ, et al. Pembrolizumabversus paclitaxel for previously treated, advanced gastric or gastro-oesophageal junction cancer (KEYNOTE-061): a randomised, open-label, controlled, Phase 3 trial. Lancet. 2018;392:123-133. doi:10.1016/S0140-6736(18)31257-1

23. Kang YK, Boku N, Satoh T, et al. Nivolumab in patients with advanced gastric or gastro-oesophageal junction cancer refractory to, or intolerant of, at least two previous chemotherapy regimens (ONO-4538-12, ATTRACTION-2): a randomised, double-blind, placebo-controlled, phase 3 trial. Lancet. 2017;390:2461-2471. doi:10.1016/S0140-6736(17)31827-5

24. Petroni G, Buqué A, Zitvogel L, et al. Immunomodulation by targeted anticancer agents. Cancer Cell. 2021;39:310-345. doi:10.1016/ j.ccell.2020.11.009

25. Masuda K, Shoji H, Nagashima K, et al. Correlation between immune-related adverse events and prognosis in patients with gastric cancer treated with nivolumab. BMC Cancer. 2019;19:974. doi:10.1186/s12885-019-6150-y

26. Barish R, Gates E, Barac A. Trastuzumab-induced cardiomyopathy. Cardiol Clin. 2019;37:407-418. doi:10.1016/j.ccl.2019.07.005

27. Nemeth BT, Varga ZV, Wu WJ, Pacher P. Trastuzumab cardiotoxicity: from clinical trials to experimental studies. Br J Pharmacol. 2017;174:3727-3748. doi:10.1111/bph.13643 


\section{Publish your work in this journal}

OncoTargets and Therapy is an international, peer-reviewed, open access journal focusing on the pathological basis of all cancers, potential targets for therapy and treatment protocols employed to improve the management of cancer patients. The journal also focuses on the impact of management programs and new therapeutic agents and protocols on patient perspectives such as quality of life, adherence and satisfaction. The manuscript management system is completely online and includes a very quick and fair peer-review system, which is all easy to use. Visit http://www.dovepress.com/ testimonials.php to read real quotes from published authors. 